\title{
Validación del cuestionario de motivación en Educación Física en educación primaria (CMEF-EP)*
}

\author{
Validation of the Motivation in Physical Education \\ Questionnaire in Primary Education (CMEF-EP)
}

Recibido: 03 de septiembre de 2012 | Aceptado: 02 de febrero de 2016

\author{
Francisco Miguel LeO ** \\ Universidad de Extremadura, España \\ José Miguel GarcíA-FernÁNDEZ **** \\ Universidad Católica San Antonio de Murcia, España \\ DAVID SÁNCHEZ-OLIVA \\ JuAN José PULIDO \\ Tomás GarcíA-CALVO \\ Universidad de Extremadura, España
}

doi : 10.11144/Javeriana.upsy15-1.vmpe

Para citar este artículo: Leo, F. M., García-Fernández, J. M., Sánchez-Oliva, D., Pulido, J. J., \& GarcíaCalvo, T. M. (2016). Validation of the Motivation in Physical Education Questionnaire in Primary Education (CMEF-EP). Universitas Psychologica, 15(1), 315-326. http://dx.doi.org/10.11144/Javeriana. upsy15-1.vmpe

* Artículo de Investigación Científica. No hubo financiación.

** Universidad Catolica de Murcia. Departamento de Didáctica de la Expresión Musical, Plástica y Corporal. Profesor contratado doctor. Correo electrónico: franmilema@unex.es

**** Universidad de Extremadura Facultad de Deporte. Correo electrónico: jmgf@ucam.edu
RESUMEN

El objetivo de la presente investigación era validar al contexto de la educación primaria el instrumento CMEF (Cuestionario de motivación en Educación Física), desarrollado originalmente en la etapa de educación secundaria. Para ello, participaron un total de 333 alumnos de educación primaria, de género masculino $(n=183)$ y femenino $(n=150)$, con edades comprendidas entre los 10 y los 12 años $(M=10.92 ; D T=0.77)$ pertenecientes a seis centros educativos públicos de la comunidad autónoma de Murcia. Los resultados obtenidos demostraron que la escala presentaba una adecuada validez factorial a partir de los índices de ajuste obtenidos en el análisis factorial confirmatorio, así como valores aceptables de consistencia interna $(\alpha>0.70)$. De la misma manera, se correlacionaron los factores del instrumento con la percepción de comportamientos positivos en la Educación Física, obteniendo una adecuada validez nomológica. Por tanto, este estudio aporta evidencias de que el Cuestionario de motivación en la Educación Física también permite analizar los tipos de regulación motivacional de los alumnos en las clases de Educación Física en la etapa de educación primaria.

Palabras clave

autodeterminación; cuestionario; propiedades psicométricas; regulaciones motivacionales

\footnotetext{
A B S T R A C T

The aim of this study was to validate in the Primary Education context the Motivational Questionnaire in Physical Education (CMEF), originally developed in Secondary Education. The sample size was formed by 333 primary school students, both male $(n=183)$ and female $(n=150)$, ranging in age from 10 to 12 years $(M=10.92, S D=0.77)$ from six public schools of the autonomous community of Murcia. Results showed that the scale had an adequate factorial validity through the fit index obtained in confirmatory factorial analysis, as well as acceptable internal consistency values $(\alpha>$ 0.70). Likewise, instruments' factors were correlated with perception of positive behaviors in physical education, obtaining an adequate nomological validity. Therefore, this study provides evidence that the Motivation in Physical Education Questionnaire also allows analysing the types of students' motivational regulation of Physical Education classes in Primary Education. Keywords self-determination; questionnaire; psychometric properties; motivational regulations
} 


\section{Introducción}

En los últimos años, a pesar de los esfuerzos de las diferentes instituciones para incentivar la práctica de actividad física (AF) y concienciar de sus beneficios entre los más jóvenes, la inactividad sigue aumentando y se está convirtiendo en uno de los problemas sociales más trascendentes de los países desarrollados (Kuehnen et al., 2012). Es por ello que la asignatura de Educación Física (EF) se ha convertido en un escenario idílico para muchos jóvenes, y único para otros, como realización alguna de AF, destacándose como un medio excelente para fomentar la motivación y la adherencia para realizar una práctica deportiva en horario extraescolar (Hagger \& Chatzisarantis, 2012; Tessier, Sarrazin, \& Ntoumanis, 2010), así como para desarrollar valores sociales y personales como la tolerancia, el respeto, la cooperación, el control y el esfuerzo (Sánchez-Oliva, Viladrich, Amado, GonzálezPonce, \& García-Calvo, 2014).

Concretamente, diferentes estudios han demostrado la enorme importancia que adquiere la motivación para explicar y predecir la práctica de actividad física extraescolar, destacándose la motivación hacia las clases de EF como un predictor positivo de la práctica de $\mathrm{AF}$ a nivel extralectivo (Barkoukis, Hagger, Lambropoulos, \& Tsorbatzoudis, 2010; González-Cutre et al., 2014). Sin embargo, analizando la bibliografía existente, existen pocas escalas encaminadas a examinar los motivos de práctica de AF en escolares en la etapa de educación primaria. Por tanto, el propósito general de este estudio es adaptar un instrumento que permita valorar el tipo de motivación de los alumnos en el ámbito de la EF en primaria.

Con base en lo anterior, una de las teorías más utilizadas para tratar de explicar el por qué una persona se involucra en una determinada actividad, es la Teoría de la autodeterminación (TAD) (Deci \& Ryan, 1985; Ryan \& Deci, 2000). Los postulados de la TAD diferencian la motivación en tres grandes bloques. El concepto de autodeterminación hace referencia a las conductas volitivas e involuntarias que presenta un sujeto a la hora de hacer cualquier tipo de actividad. Ordenados de mayor a menor grado de autodeterminación, en el primer bloque se encuentra la motivación intrínseca, caracterizada porque la persona guía su comportamiento por el mero placer y satisfacción que le produce participar en lo que hace. En el segundo nivel se encuadra la motivación extrínseca, subdividida a su vez en diferentes regulaciones: en primer lugar, se encuentra la regulación integrada, definida por la introversión de la actividad en el día a día de la persona, considerándose como una parte más de su cotidianeidad. Este tipo de regulación no es característica en niños y adolescentes, debido a que no tienen definidos sus rasgos personales en su totalidad (Vallerand \& Rosseau, 2001). Seguidamente, la regulación identificada se caracteriza porque una persona realiza una actividad tras valorar los beneficios que le reporta, a nivel social y personal. A continuación, la regulación introyectada se caracteriza por el sentimiento de culpabilidad que aparece en el sujeto cuando no llega a realizarse la actividad prevista, o por la ejecución por orgullo como protagonista que incentiva llevarla a cabo.

Posteriormente, en el último nivel dentro de la motivación extrínseca, se encuadra la regulación externa, en la cual el comportamiento está determinado por la consecución de alguna recompensa a cambio: éxito, reconocimiento, premios, etc. Por último, en el menor grado de autodeterminación se encuentra la desmotivación, caracterizada por la total ausencia de regulaciones intrínsecas y extrínsecas para regular la conducta hacia la realización de cualquier actividad, y no encontrado sentido a seguir haciéndola.

Así pues, a lo largo del desarrollo de este postulado han sido diseñadas y validadas diversas escalas que han tratado de determinar el tipo de regulación motivacional en diferentes contextos: laboral (Gagné et al., 2010), deportivo (Pelletier et al., 1995; Lonsdale, Hodge, \& Rose, 2008) o académico. Centrándonos en el ámbito escolar, muchos estudios han decidido adaptar cuestionarios desarrollados previamente en el deporte, como es el caso de la adaptación de la Sport Motivation Scale (SMS) (Pelletier et al., 1995) al contexto de la EF (Boiché, Sarrazin, Grouzet, Pelletier, \& Chanal, 
2008; Moreno, González-Cutre, \& Chillón, 2009; Radel, Sarrazin, Legrain, \& Wild, 2010; Standage, Duda, \& Ntoumanis, 2003; Zahariadis, Tsorbatzoudis, \& Grouios, 2005). Este instrumento está compuesto por un total de 28 ítems, los cuales subdividen la motivación intrínseca en tres subfactores: motivación para conocer, para experimentar y para lograr. Sin embargo, este instrumento ha presentado alguna limitación, como son los valores discretos obtenidos en los índices de fiabilidad, por lo que los propios autores decidieron desarrollar en el ámbito deportivo la SMS - II (Pelletier, Rocchi, Vallerand, Deci, \& Ryan, 2013).

Del mismo modo, muchas investigaciones han optado por utilizar la Escala del locus percibido de causalidad (PLOC) desarrollada por Goudas, Biddle \& Fox (1994), instrumento creado a partir del Cuestionario de autorregulación de Ryan \& Conell (1989), en el que añadieron el factor desmotivación de la Escala de motivación académica (Vallerand et al., 1992), conformando una escala compuesta por 20 ítems (cuatro por factor). Esta escala fue traducida y validada en el contexto español por Moreno et al. (2009), y ha sido ampliamente utilizada en diferentes estudios. Respecto a las propiedades psicométricas de este cuestionario, Vlachopoulos, Katartzi, Kontou, Moustaka, \& Goudas (2011) realizaron una revisión sobre los trabajos que habían utilizado el PLOC para analizar el tipo de motivación en las clases de EF, encontrándose cómo en la mayoría de los estudios analizados, los valores de consistencia interna no eran adecuados en todos los factores del cuestionario, motivo por el cual desarrollaron una versión revisada del Perceived Locus of Causality (PLOC- R) con chicos y chicas de $5^{\circ}$ y $6^{\circ}$ de primaria, registrando valores adecuados en cuento a las propiedades psicométricas del instrumento.

A tenor de los resultados reflejados en la revisión realizada por Vlachopoulos et al. (2011), el desarrollo de esta escala en el ámbito educativo ha presentado ciertas limitaciones, derivadas en algunas ocasiones de la traducción y su posterior aplicación con muestras con características culturales, educativas, socioeconómicas y cronológicas diferentes. Ante esta situación, Sánchez-Oliva, Leo, Amado,
González-Ponce y García-Calvo (2012) elaboraron un nuevo cuestionario para tratar de valorar el tipo de regulación de los alumnos de secundaria durante las clases de EF, denominado Cuestionario de motivación en Educación Física. Esta escala se compone de 20 ítems que examinan la motivación intrínseca, así como las diferentes regulaciones extrínsecas (excepto la integrada, al ser adolescentes) y la desmotivación, presentando además adecuadas propiedades psicométricas con una muestra formada por estudiantes de secundaria.

Sin embargo, a la hora de implementar con alumnos de primaria un cuestionario creado para alumnos de educación secundaria, hay que tener en cuenta las diferencias existentes en cuanto al nivel de maduración, lo que puede provocar que el mismo cuestionario no opere de la misma forma en alumnos de primaria y secundaria. Por tanto, teniendo en cuenta las diferencias existentes entre estos alumnos, el principal objetivo del estudio era comprobar las propiedades psicométricas del Cuestionario de motivación en Educación Física en una etapa más temprana, como la educación primaria. Además, se pretendía examinar la validez nomológica del instrumento, para lo que se han incluido los factores del Cuestionario de comportamientos positivos en Educación Física (SánchezOliva, Sánchez-Miguel, Leo, Amado, y GarcíaCalvo, 2013b). Derivada del principal objetivo del estudio, la hipótesis general que se plantea es que el CMEF-EP presentará valores adecuados de consistencia interna, fiabilidad y una adecuada validez nomológica, correlacionándose positivamente con la percepción de esfuerzo, control, cooperación, tolerancia y respeto.

\section{Método}

\section{Participantes}

La muestra del estudio estaba formada por un total de 333 alumnos de EF, de género masculino $(n=$ $183)$ y femenino $(n=150)$, con edades comprendidas entre los 10 y los 12 años $(M=10.92$; DT $=0.77$ ) pertenecientes a seis centros educativos públicos de la comunidad autónoma de Murcia. 
Los participantes eran del último ciclo de primaria, distribuidos en dos cursos, quinto $(n=151)$ y sexto $(n=182)$. El porcentaje de muestra que se eliminó no superó el 4\%, con una tasa de participación del $96.1 \%$, con sólo 12 cuestionarios invalidados (3.87\%) de un total de 389 cuestionarios recogidos.

\section{Instrumentos]}

Motivación. Se empleó el Cuestionario de motivación en la Educación Física (CMEF) (SánchezOliva et al., 2012). Esta escala está compuesta por la frase inicial "Yo participo en las clases de Educación Física...”, seguida de 20 ítems que analizan los cinco factores, entre ellos: respecto a la motivación intrínseca (4 ítems, ej.: "Porque la Educación Física es divertida”), regulación identificada (4 ítems; ej.: "Porque esta asignatura me aporta conocimientos y habilidades que considero importantes"), regulación introyectada (4 ítems; ej.: "Porque lo veo necesario para sentirme bien conmigo mismo"), regulación externa (4 ítems: ej.: "Para demostrar al profesor/a y compañeros/ as mi interés por la asignatura") y desmotivación (4 ítems; ej.: "Pero realmente siento que estoy perdiendo mi tiempo con esta asignatura"). Los participantes debían expresar su grado de acuerdo utilizando una escala tipo Likert con cinco opciones de respuesta, desde totalmente en desacuerdo (1) a totalmente de acuerdo (5) con la formulación de la frase.

Comportamientos positivos en Educación Física. Para valorar la percepción que el alumnado tenía sobre el desarrollo de comportamientos positivos en las clases de EF, se utilizó el Cuestionario de comportamientos positivos en Educación Física (CCPEF) (Sánchez-Oliva et al., 2013b). Este cuestionario en su versión original se presenta de forma dicotómica, pero por razones de simplificación, en el presente estudio solamente se ha utilizado la vertiente positiva de cada ítem. El instrumento está compuesto por la frase inicial "En las clases de Educación Física...", seguida de 18 ítems que analizan los cinco factores incluidos: respeto a las normas, instalaciones y materiales (4 ítems; ej.: "Respeto las instalaciones del centro”), valoración del esfuerzo
(3 ítems; ej.: "Lo más importante es esforzarse al máximo"), tolerancia y respeto a los demás, (4 ítems; ej.: "Soy tolerante con la actuación de mis compañeros"), cooperación (3 ítems; ej.: "Me encanta participar en trabajos de grupo") y autocontrol (4 ítems; ej.: "Cuando se me acaba la paciencia, sé controlar mis impulsos"). Los participantes debían expresar su grado de acuerdo utilizando una escala tipo Likert con cinco opciones de respuesta, desde totalmente en desacuerdo (1) a totalmente de acuerdo (5) con la formulación de la frase.

\section{Procedimiento}

En primer lugar, la investigación fue aprobada por la Comisión Ética de la Universidad de Extremadura, cumpliendo las normas establecidas en la Declaración de Helsinki de 1964 y con las exigencias éticas que marcan la American Psychological Association (APA, 2009). Se llevó a cabo un diseño transversal, realizándose una medida a mitad del curso académico para dejar el tiempo suficiente de asimilación con el curso, asignatura y profesorado. Para llevar a cabo la recogida de datos, se desarrolló un protocolo de actuación para que la obtención de datos fuera similar en todos los participantes. Se informó a los docentes sobre cuáles eran los objetivos de la investigación y cuál iba a ser la utilización de los resultados. Asimismo, se diseñó una carta de consentimiento para directores y otra para los tutores de los menores participantes, quienes debían devolverla firmada para autorizar la colaboración en el estudio.

Una vez obtenidos los permisos y el consentimiento informado, se procedió a la toma de datos. Los participantes rellenaron los cuestionarios en una clase en el horario de EF, de manera individual y en un clima adecuado que les permitía concentrarse sin tener ningún tipo de distracción. A su vez, se contó con la ayuda de uno de los investigadores, que estuvo presente en la recogida de datos y prestó la atención necesaria ante cualquier duda o imprevisto que surgieran entre los participantes. Por último, el proceso de realización tuvo una duración aproximada de entre 10-12 minutos. 


\section{Análisis de datos}

Para el tratamiento de los datos se utilizaron los programas estadísticos SPSS 19.0 y Amos 19.0. Con el objetivo de valorar las propiedades psicométricas, se llevó a cabo un análisis factorial exploratorio (AFE) con componentes principales y rotación varimax para confirmar la distribución de los ítems en cada uno de los factores en educación primaria. A continuación, se desarrolló un análisis factorial confirmatorio (AFC) con el Método de estimación de parámetros de máxima verosimilitud siguiendo las recomendaciones de Merenda (2007), utilizando los siguientes índices para verificar el ajuste del modelo: $\chi^{2} / \mathrm{gl}$ (Chi-Cuadrado/Grados de libertad), SRMR (Standardized Root Mean Residual), RMSEA (Root Mean Square Error of Approximation), CFI (Comparative Fit Index), TLI (Tuker-Lewis Index) y GFI (Goodness of Fit Index). Además, se analizaron los estadísticos descriptivos, los valores de normalidad (asimetría y curtosis) y los índices de fiabilidad (alfa de Cronbach) de cada uno de los factores que componen el instrumento. Asimismo, se examinó la validez discriminante y nomológica del instrumento por medio del análisis de correlación bivariada, para comprobar el grado de asociación entre los factores que componen el CMEF y los factores del CCPEF.

\section{Resultados}

\section{Propiedades psicométricas del CMEF en educación primaria}

En primer lugar, se calculó el valor de la medición de adecuación muestral de Kaiser-Meyer-Olkin (KMO), obteniendo de un valor adecuado (0.86) conforme a lo expuesto por Kaiser (1974). El test de esfericidad de Bartlett fue también significativo $(p<0.01)$, lo que indica la adecuación de los datos.

En segundo lugar, se examinaron los patrones de distribución y los componentes subyacentes de los 20 ítems, a través de un análisis de componentes principales. Stevens (2002) sugirió que este tipo de análisis es apropiado cuando se desea que las varia- bles (es decir, los ítems) sean libres de asociarse con todos los componentes y que uno de los objetivos del análisis es determinar el número de componentes subyacentes. Las consideraciones y criterios comunicados por Stevens se utilizaron en el estudio actual para guiar las decisiones tomadas acerca de la idoneidad de los artículos. A su vez, se aplicó una rotación ortogonal varimax (Kaiser, 1958) para facilitar la interpretación de los componentes.

Todos los ítems obtuvieron cargas factoriales superiores a 0.60 lo que indica una estructura factorial compuesta por cinco factores. Sin embargo, hay que tener en cuenta que el ítem tres y 13 obtuvieron un factor de carga elevado en dos factores diferentes, regulación introyectada e identificada $(>0.37)$, por lo que se decidió eliminar ambos ítems. Por otro lado, obtuvimos autovalores superiores a uno, y una varianza total explicada del $54.77 \%$.

\section{Análisis factorial confirmatorio}

Con el fin de ratificar la estructura factorial resultante del AFE, en el estudio se comprobó la estructura de primer orden con los cinco factores tal y como señalan (Byrne, 2008). Para ello, hemos llevado a cabo un AFC, usando el método de estimación de máxima verosimilitud con el procedimiento de bootstrapping, que aseguraban que los resultados de las estimaciones eran robustos y, por lo tanto, no se ven afectados por la falta de normalidad multivariante (Byrne, 2001). Como comentamos con anterioridad, hemos utilizado los siguientes índices de ajuste para evaluar la adecuación de los datos al modelo: $\chi^{2} / \mathrm{gl}$ (Chi-Cuadrado / Grados de libertad), SRMR (Standardized Root Mean Residual), RMSEA (Root Mean Square Error of Approximation), CFI (Comparative Fit Index), TLI (Tuker-Lewis Index) y GFI (Goodness of Fit Index). En el caso de $\chi^{2} / d f$, los valores inferiores a tres se consideraron aceptables (Bentler, 1989). Hu y Bentler (1999) afirman que las puntuaciones superiores a 0.90 para los índices incrementales como CFI e IFI pueden ser aceptables, y valores superiores a 0.95 se consideran excelentes. Además, el modelo se estima que tiene un buen ajuste si el RMSEA es inferior a 0.06 y SRMR es inferior a 0.08 (Browne 
Tabla 1.

Análisis factorial exploratorio y validez convergente

\begin{tabular}{|c|c|c|c|c|c|}
\hline & Intrínseca & Identificada & Introyectada & Externa & Desmotivación \\
\hline 1. Porque la Educación Física es divertida & 0.70 & 0.14 & 0 & 0.11 & 0.08 \\
\hline $\begin{array}{l}\text { 6. Porque esta asignatura me resulta agradable } \\
\text { e interesante }\end{array}$ & 0.65 & 0.27 & 0.11 & -0.01 & -0.12 \\
\hline $\begin{array}{l}\text { 11. Porque me lo paso bien realizando las } \\
\text { actividades }\end{array}$ & 0.75 & 0.07 & 0.01 & 0.06 & -0.25 \\
\hline 16. Por la satisfacción que siento al practicar. & 0.63 & 0.27 & 0.22 & 0.18 & -0.23 \\
\hline $\begin{array}{l}\text { 2. Porque puedo aprender habilidades que } \\
\text { podría usar en otras áreas de mi vida }\end{array}$ & -0.01 & 0.72 & 0.04 & 0.05 & -0.04 \\
\hline $\begin{array}{l}\text { 7. Porque valoro los beneficios que puede } \\
\text { tener esta asignatura para desarrollarme como } \\
\text { persona. }\end{array}$ & 0.28 & 0.61 & -0.08 & 0.10 & 0.04 \\
\hline $\begin{array}{l}\text { 12. Porque, para mí, es una de las mejores } \\
\text { formas de conseguir capacidades útiles para } \\
\text { mi futuro }\end{array}$ & 0.16 & 0.64 & 0.10 & 0.21 & -0.12 \\
\hline $\begin{array}{l}\text { 17. Porque esta asignatura me aporta } \\
\text { conocimientos y habilidades que considero } \\
\text { importantes }\end{array}$ & 0.16 & 0.72 & 0.09 & 0.04 & -0.16 \\
\hline $\begin{array}{l}\text { 3. Porque es lo que debo hacer para sentirme } \\
\text { bien }\end{array}$ & 0.25 & 0.39 & 0.47 & $=0.08$ & 0.13 \\
\hline $\begin{array}{l}\text { 8. Porque me siento mal si no participo en las } \\
\text { actividades }\end{array}$ & -0.04 & 0.01 & 0.79 & 0.19 & 0.13 \\
\hline $\begin{array}{l}\text { 13. Porque lo veo necesario para sentirme } \\
\text { bien conmigo mismo }\end{array}$ & 0.24 & 0.37 & 0.45 & 0.20 & $=0.01$ \\
\hline $\begin{array}{l}\text { 18. Porque me siento mal conmigo mismo si } \\
\text { falto a clase }\end{array}$ & 0.03 & 0.01 & 0.74 & 0.23 & -0.01 \\
\hline $\begin{array}{l}\text { 4. Porque está bien visto por el profesor y los } \\
\text { compañeros. }\end{array}$ & 0.01 & 0.05 & 0.06 & 0.66 & 0.28 \\
\hline $\begin{array}{l}\text { 9. Porque quiero que el profesor/a piense que } \\
\text { soy un/a buen/a estudiante }\end{array}$ & 0.09 & 0.03 & 0.12 & 0.84 & 0.06 \\
\hline $\begin{array}{l}\text { 14. Porque quiero que mis compañeros/as } \\
\text { valoren lo que hago. }\end{array}$ & 0.06 & 0.09 & 0.26 & 0.73 & 0.04 \\
\hline $\begin{array}{l}\text { 19. Para demostrar al profesor/a y } \\
\text { compañeros/as mi interés por la asignatura. }\end{array}$ & 0.10 & 0.21 & 0.12 & 0.74 & 0.01 \\
\hline $\begin{array}{l}\text { 5. Pero no comprendo por qué debemos tener } \\
\text { Educación Física }\end{array}$ & 0.05 & -0.12 & -0.10 & 0.15 & 0.61 \\
\hline $\begin{array}{l}\text { 10. Pero realmente siento que estoy } \\
\text { perdiendo mi tiempo con esta asignatura. }\end{array}$ & -0.12 & -0.04 & 0.11 & 0.07 & 0.72 \\
\hline $\begin{array}{l}\text { 15. No lo sé, tengo la impresión de que es } \\
\text { inútil seguir asistiendo a clase }\end{array}$ & -0.01 & 0.01 & 0.06 & 0.07 & 0.70 \\
\hline $\begin{array}{l}\text { 20. No lo sé claramente porque no me gusta } \\
\text { nada. }\end{array}$ & -0.26 & -0.07 & 0.06 & 0.01 & 0.71 \\
\hline Varianza explicada & 6.13 & 14.41 & 5.29 & 21.77 & 7.17 \\
\hline Autovalor & 1.23 & 2.88 & 1.06 & 4.35 & 1.43 \\
\hline
\end{tabular}

Fuente: elaboración propia

y Cudeck, 1993). En este sentido, los valores de los índices de ajuste en todos los casos fueron aceptables: $\chi^{2} / \mathrm{gl}=1.80 ; \mathrm{CFI}=0.92 ; \mathrm{TLI}=0.91 ; \mathrm{GFI}=$ $0.93 ;$ SRMR $=0.05$ y RMSEA $=0.04$.
Estadísticos descriptivos, normalidad, consistencia interna y validez nomológica

La Tabla 2 presenta los estadísticos descriptivos 


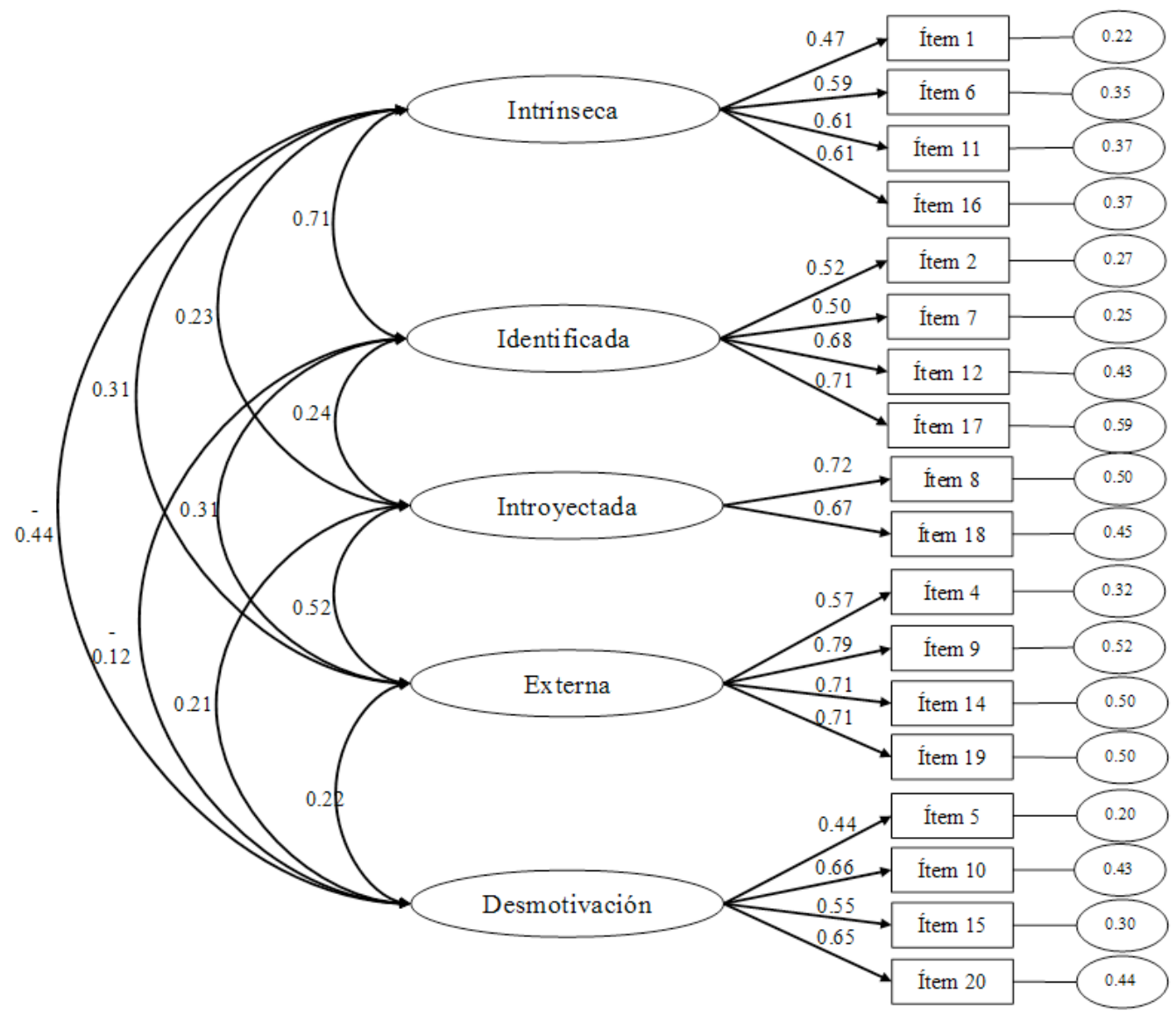

Figura 1. Análisis Factorial Confirmatorio CMEF-EP

Fuente: elaboración propia

de los factores de motivación, donde se observan que las medias están por encima de los valores centrales en la motivación intrínseca y la regulación identificada, mientras que la regulación introyectada y externa presentan medias con valores centrales. En cambio, la desmotivación presenta medias bajas. Asimismo, en esta tabla se pueden observar los valores de asimetría y curtosis, así como la consistencia interna de todos los factores, donde se observan puntuaciones entorno a 0.70 (Nunnally \& Bernstein, 1994), por lo que se pueden considerar aceptables (Hair, Anderson, Tatham, \& Black, 1998).

Para confirmar la validez nomológica, se introdujo la percepción de comportamientos positivos en educación física, ya que es una de las variables que ha sido más estrechamente asociada a la motiva- ción (Sánchez-Oliva et al., 2014). En este sentido, los factores de motivación intrínseca y la regulación identificada en mayor medida, y la regulación introyectada y la regulación externa mostraron correlaciones positivas con percepción de comportamientos positivos. En cambio, la desmotivación se relacionó de forma negativa en todos los casos, como anteriormente habían señalado otros autores (Sánchez-Oliva et al., 2014).

\section{Discusión}

A través del presente estudio se pretendían analizar las propiedades psicométricas del CMEF (SánchezOliva et al., 2012) en una muestra formada por alumnos del tercer ciclo de educación primaria. Previamente, este cuestionario había sido validado 
Francisco Miguel Leo, José Miguel García-Fernández, David SÁnchez-

Oliva, Juan José Pulido, Tomás García-Calvo

TABLA 2.

Estadísticos descriptivos, normalidad, consistencia interna y validez nomológica

\begin{tabular}{lcccccccccc}
\hline & $\mathrm{M}$ & $\mathrm{SD}$ & Asimetría & Kurtosis & $\alpha$ & 1 & 2 & 3 & 4 & 5 \\
\hline Intrínseca & 4.62 & 0.55 & -2.13 & 6.16 & 0.65 & $0.12^{*}$ & $0.31^{* *}$ & $0.34^{* *}$ & $0.20^{* *}$ & $0.30^{* *}$ \\
Identificada & 4.49 & 0.62 & -1.70 & 3.83 & 0.69 & $0.22^{* *}$ & $0.33^{* *}$ & $0.34^{* *}$ & $0.38^{* *}$ & $0.39^{* *}$ \\
Introyectada & 3.64 & 0.99 & -0.43 & -0.57 & 0.65 & 0.06 & $0.21^{* *}$ & $0.20^{* *}$ & $0.23^{* *}$ & $0.23^{* *}$ \\
Externa & 3.60 & 1.18 & -0.56 & -0.81 & 0.78 & -0.01 & $0.11^{*}$ & $0.21^{* *}$ & $0.15^{* *}$ & $0.16^{* *}$ \\
Desmotivación & 1.50 & 0.81 & 1.82 & 3.03 & 0.64 & $-0.22^{* *}$ & $-0.25^{* *}$ & $-0.17^{* *}$ & $-0.14^{* *}$ & -0.10 \\
\hline
\end{tabular}

Nota: 1) respeto a las instalaciones; 2) cooperación; 3) valoración del esfuerzo; 4) autocontrol; 5) respeto a los compañeros.

$* p<0.05, * * p<0.01$

Fuente: elaboración propia

eficazmente con alumnos de secundaria. Sin embargo, debido a las diferencias personales y sociales que pueden existir en los adolescentes en función del ciclo educativo en el que se encuentran, entendíamos que la validación de este instrumento con alumnos de primaria podía ofrecer a la comunidad científica una información más completa acerca de la idoneidad de esta escala para valorar el tipo de regulación motivacional en el contexto de la EF.

Con el objetivo de analizar las propiedades psicométricas del instrumento, en primer lugar, se realizó un AFE, lo que permite conocer el grado de asociación saturación de cada uno de los ítems sobre los diferentes factores incluidos. Como ha sido explicado en el apartado de resultados, todos los ítems obtuvieron excelentes puntuaciones (>0.60), a excepción de los indicadores tres y 13 . Este hecho, sumado a que dichos ítems también obtuvieron cargas factoriales elevada $(>0.35)$ con respecto al factor 2 (regulación identificada), propició la eliminación de estos dos ítems a la hora de realizar los siguientes análisis. Con esto, se pone de manifiesto cómo los alumnos de primaria también entendieron estos dos ítems como razones identificadas para involucrarse en las clases de EF. De esta forma, quizás las frases "es lo que debo hacer" y "lo veo necesario" pudieron ser entendidos por los alumnos como beneficios asociados a la actividad y, por lo tanto, lo asocian a la regulación identificada.

Tras el AFE, el siguiente paso suponía confirmar la estructura resultante en el análisis anterior, a través de un AFC. Para ello, se incluyeron los 18 ítems que conformaban el cuestionario, a excepción de los ítems tres y 13. Respecto a los pesos de regresión obtenidos por los diferentes ítems con sus factores correspondientes, todos los indicadores registraron valores superiores a 0.40 (Brown, 2006). Además, la estructura factorial mostró un adecuado ajuste global a los datos, lo que ratifica la validez factorial de la estructura resultante en el AFE.

En cuanto a las relaciones obtenidas entre los cinco factores que componen el cuestionario, la motivación intrínseca se relacionó positivamente con las regulaciones identificada, introyectada y externa, y de forma negativa con la desmotivación. En el caso de la desmotivación, se obtuvo una relación positiva con las regulaciones externa e introyectada y una relación negativa con la regulación identificada. En líneas generales, estos resultados son consistentes con los encontrados en la validación inicial del CMEF (Sánchez-Oliva et al., 2012) y están en línea con los postulados expuestos en la TAD (Deci \& Ryan, 2000; Ryan $\&$ Deci, 2000).

No obstante, especial atención requiere la relación positiva obtenida entre la regulación intrínseca y la regulación externa. Según exponen los autores de referencia para esta teoría (Deci \& Ryan, 2000; Ryan \& Deci, 2000), esta asociación debería ser negativa, aspecto que también ha sido constatado en la validación de algunas escalas encaminadas a analizar el tipo de motivación en las clases de EF (Moreno et al., 2009; Vlachopoulos et al., 2011). Sin embargo, en los últimos años se encontraron estudios que han mostrado una relación positiva entre las regulacio- 
nes intrínseca y externa, como el desarrollado por Cecchini, Fernández-Losa, González, y Cecchini (2013) en EF en la etapa de primaria u otros estudios desarrollados tanto el contexto deportivo (Bara et al., 2011; Moreno, Cervelló, y González-Cutre, 2006, 2007) como en el ámbito de la AF (Sebire, Jago, Fox, Edwards, \& Thompson, 2013). En el caso del cuestionario que aquí se presenta, analizando los ítems que componen las regulaciones intrínseca y externa, el motivo de esta relación positiva puede estar en que existen alumnos que estando motivados intrínsecamente hacia las clases de EF (niveles altos de satisfacción, disfrute, placer...), también se involucran en las clases de EF por otros motivos externos, como el hecho de agradar al profesor y los compañeros. No obstante, son necesarios más estudios encaminados a confirmar el sentido de la relación entre las regulaciones intrínseca y externa.

El siguiente objetivo del estudio suponía analizar la validez nomológica del instrumento, a través de un análisis correlacional con diferentes comportamientos positivos. Como ha sido explicado en el apartado de resultados, tanto la motivación intrínseca como los tipos de regulaciones extrínsecas se relacionaron positivamente con los comportamientos positivos, de forma que a medida que desciende el nivel de autodeterminación, también lo hace el coeficiente de correlación.

En el caso de los niveles altos de autodeterminación, estos resultados son consistentes con estudios previos (Durão, 2008; Sánchez-Oliva, Leo, SánchezMiguel, Amado, \& Garcia-Calvo, 2013a; SánchezOliva et al., 2014), evidenciando con ello el hecho de que aquellos alumnos que presentan una motivación autodeterminada son los que desarrollan un mayor número de comportamientos adaptativos (respeto, tolerancia, autocontrol, cooperación...). Con respecto a la regulación externa, los resultados están en línea con los encontrados en el trabajo de Sánchez-Oliva et al. (2014), sin embargo, no son consistentes con el trabajo de Durão (2008), por lo que son necesarios más estudios encaminados a comprobar las consecuencias provocadas por la regulación externa.

Con respecto a la desmotivación, esta variable se relacionó negativamente con los cinco comportamientos positivos, ratificándose los postulados expuestos en la TAD y en línea con estudios previos (Durão, 2008; Sánchez-Oliva et al., 2014), destacándose cómo aquellos alumnos que se sienten desmotivados hacia la EF son los que desarrollan menos comportamientos positivos, quizás debido a la aparición de sentimientos como la frustración o el aburrimiento, que pueden desembocar en la realización de conductas desadaptativas durante las clases de EF.

\section{Conclusiones, limitaciones y futuras líneas de investigación}

A modo de conclusión, el presente estudio ha demostrado las propiedades psicométricas del CMEF con alumnos de primaria, lo que permite utilizar eficazmente este instrumento con alumnos de estas edades. En el último grado de primaria, los alumnos comienzan a tener conciencia de "por qué" se involucran en una determinada actividad. En este sentido, el presente estudio propone una herramienta para conocer los motivos por los cuales los alumnos de primaria se involucran en las clases de EF y, a partir de ahí, conocer la incidencia de los diferentes tipos de motivación sobre importantes consecuencias, como pueden ser la práctica de actividad física a nivel extraescolar.

En cuanto a las limitaciones del estudio, quizás el tamaño muestral no es elevado, teniendo en cuenta las numerosas variables incluidas en el modelo factorial. Además, la relación positiva encontrada entre la motivación intrínseca y la regulación externa también supone una limitación en el estudio y anima a los investigadores a esclarecer la asociación entre estas dos variables. Del mismo modo, como futuras líneas de investigación, sería interesante poder adaptar esta escala a otros contextos como el deporte o la AF y comprobar así la eficacia de esta escala para valorar el tipo de motivación en dichos contextos.

\section{Referencias}

American Psychological Association (2009). Publication Manual of the American Psychological Association, Sixth Edition. 
Bara, M., Andrade, D., Miranda, R., Núñez, J. L., Martín-Albo, J., \& Ribas, P. R. (2011). Preliminary validation of a brazilian version of the Sport Motivation Scale. Universitas Psychologica, 2, 557-566.

Barkoukis, V., Hagger, M. S., Lambropoulos, G., \& Tsorbatzoudis, H. (2010). Extending the trans-contextual model in physical education and leisure-time contexts: examining the role of basic psychological need satisfaction. The British Journal of Educational Psychology, 80, 647-70.

Boiché, J. C. S., Sarrazin, P. G., Grouzet, F. M. E., Pelletier, L. G. \& Chanal, J. P. (2008). Students' motivational profiles and achievement outcomes in physical education: A self-determination perspective. Journal of Educational Psychology, 100, 688-701.

Browne, M. W., \& Cudeck, R. (1993). Alternative ways of assessing model fit. En K. A. Bollen, \& J. S. Long (Eds.), Testing Structural Equation Models (pp. 136-162). Beverly Hills, CA: Sage.

Byrne, B. M. (2001). Structural equation modeling: Perspectives on the present and the future. International Journal of Testing, 1, 327-334.

Byrne, B. M. (2008). Testing for multigroup equivalence of a measuring instrument: A walk through the process. Psichothema, 20, 872-882.

Cecchini, A. J., Fernández-losa, J. L., González, C., \& Cecchini, C. (2013). Aplicaciones del modelo de autodeterminación en la educación física de primaria. Revista Latinoamericana de Psicología, 45, 97-109.

Cheung, G. W., \& Rensvold, R. B. (2002). Evaluating goodness-of-fit indices for testing measurement equivalence. Structural Equation Modeling. A Multidisciplinary Journal, 9, 233-255.

Deci, E. L., \& Ryan, R. M. (1985). Intrinsic motivation and self-determination in human behavior. New York: Plenum Press.

Deci, E. L., \& Ryan, R. M. (2000). The "What" and "Why" of goal pursuits: Human needs and the self-determination of behavior. Psychological Inquiry, 11, 227-268.

Durão, L. M. (2008). Estudo da motivação e suas consequências comportamentais em jovens estudantes de educação física em portugal. Tesis Doctoral. Universidad de Extremadura.
Gagné, M., Forest, J., Gilbert, M. H., Aubé, C., Morin, E., \& Malorni, A. (2010). The Motivation at Work Scale: Validation evidence in two languages. Educational and Psychological Measurement, 70, 628-646.

González-Cutre, D., Ferriz, R., Beltrán-Carrillo, V. J., Andrés-Fabra, J. A., Montero-Carretero, C., Cervelló, E., \& Moreno-Murcia, J. A. (2014). Promotion of autonomy for participation in physical activity: a study based on the trans-contextual model of motivation. Educational Psychology, 34, 367-384.

Goudas, M., Biddle, S. J. H., \& Fox, K. R. (1994). Perceived locus of causality, goal orientations, and perceived competence in school physical education classes. British Journal of Educational Psychology, 64, 453-463.

Hair, J. F., Anderson, R. E., Tatham, R. L., \& Black, W. C. (1998). Multivariate Data Analysis. Upper Saddle River, NJ: Prentice Hall.

Hagger, M. S., \& Chatzisarantis, N. L. D. (2012). Transferring motivation from educational to extramural contexts: a review of the trans-contextual model. European Journal of Psychology of Education, 27, 195-212.

Hu, L., \& Bentler, P. M. (1999). Cutoff criteria for fit indexes in covariance structure analysis: Conventional criteria versus new alternatives. Structural Equation Modeling, 6, 1-55.

Kaiser, H. F. (1958). The varimax criterion for analytic rotation in factor analysis. Psychometrika, 23, 187 200.

Kaiser, H. F. (1974). An index of factorial simplicity. Psychometrika, 39, 31-36.

Kuehnen, P., Mischke, M., Wiegand, S., Sers, C., Horsthemke, B., Lau, S., \& Krude, H. (2012). An Alu element-associated hypermethylation variant of the POMC gene is associated with childhood obesity. PLoS Genetics, 8(3), e1002543.

Lonsdale, C., Hodge, K., \& Rose, E. A. (2008). The Behavioral Regulation in Sport Questionnaire (BRSQ): instrument development and initial validity evidence. Journal of Sport and Exercise Psychology, 30, 323-355.

Moreno, J. A., Cervelló, E., \& González-Cutre, D. (2006). Motivación autodeterminada y flujo dis- 
posicional en el deporte. Anales de Psicología, 22, $310-317$.

Moreno, J. A., Cervelló, E., \& González-Cutre, D. (2007). Analizando la motivación en el deporte: Un estudio a través de la teoría de la autodeterminación. Anales de Psicología, 25, 35-52.

Moreno, J. A., González-Cutre, D., \& Chillón, M. (2009). Preliminary validation in Spanish of a scale designed to measure motivation in physical education classes: The Perceived Locus of Causality (PLOC) Scale. The Spanish Journal of Psychology, 12, 327-337.

Nunnally, J. C., \& Bernstein, I. H. (1994). Psychometric theory (3rd ed.). New York, NY: McGraw-Hill.

Pelletier, L. G., Fortier, M. S., Vallerand, R. J., Tuson, K. M., Briere, N. M., \& Blais, M. R. (1995). Toward a new measure of intrinsic motivation, extrinsic motivation, and amotivation in sports: The Sport Motivation Scale (SMS). Journal of Sport and Exercise Psychology, 17, 35-53.

Pelletier, L. G., Rocchi, M. A., Vallerand, R. J., Deci, E. L., \& Ryan, R. M. (2013). Validation of the revised sport motivation scale (SMS-II). Psychology of Sport and Exercise, 14(3), 329-341.

Radel, R., Sarrazin, P., Legrain, P. \& Wild, T. C. (2010). Social contagion of motivation between teacher and student: Analyzing underlying processes. Journal of Educational Psychology, 102, 577-587.

Ryan, R. M., \& Connell, J. P. (1989). Perceived locus of causality and internalization: Examining reasons for acting in two domains. Journal of Personality and Social Psychology, 57, 749-761.

Ryan, R. M., \& Deci, E. L. (2000). Self-determination theory and the facilitation of intrinsic motivation, social development and wellbeing. The American Psychologist, 55, 68-78.

Sánchez-Oliva, D., Leo, F. M, Amado, D., GonzálezPonce, I., \& García-Calvo, T. (2012). Desarrollo de un cuestionario para valorar la motivación en educación física. Revista Iberoamericana de Psicología del Ejercicio y Deporte, 7, 227-250.

Sánchez-Oliva, D., Leo, F. M., Sánchez-Miguel, P. A., Amado, D., \& García-Calvo, T. (2013a). Desarrollo de un modelo causal para explicar los comportamientos positivos en las clases de Educación Física. Acción Motriz, 10, 48-58.
Sánchez-Oliva, D., Sánchez-Miguel, P. A., Leo, F. M., Amado, D., \& García-Calvo, T. (2013b). Desarrollo y validación de un cuestionario para analizar la percepción de comportamientos positivos en las clases de educación física. Cultura y Educación, 25, 495-507.

Sánchez-Oliva, D., Viladrich, C., Amado, D., GonzálezPonce, I., \& García-Calvo, T. (2014). Prediction of positive behaviors in physical education: A self-determination theory perspective. Journal of Psychodidactics, 19, 387-406.

Sebire, S. J., Jago, R., Fox, K. R., Edwards, M. J., \& Thompson, J. L. (2013). Testing a self-determination theory model of children's physical activity motivation: a cross-sectional study. The International Journal of Behavioral Nutrition and Physical Activity, 10, 111.

Standage, M., Duda, J. L., \& Ntoumanis, N. (2003). A model of contextual motivation in physical education: Using constructs from self-determination and achievement goal theories to predict physical activity intentions. Journal of Educational Psycho$\log y$, 95, 97-110.

Stevens, J. P. (2002). Applied Multivariate Statistics for the Social Sciences. Mahwah, NJ: Lawrence Erlbaum Associates.

Tessier, D., Sarrazin, P., \& Ntoumanis, N. (2010). The effect of an intervention to improve newly qualified teachers' interpersonal style, students motivation and psychological need satisfaction in sport-based physical education. Contemporary Educational Psychology, 35, 242-253.

Vallerand, R. J., Pelletier, L. G., Blais, M. R., Briere, N. M., Senecal, C., \& Vallieres, E. F. (1992). The academic motivation scale: A measure of intrinsic, extrinsic, and amotivation in education. Education and Psychological Measurement, 52, 1003-1017.

Vallerand, R. J., \& Rousseau, F. L. (2001). Intrinsic and extrinsic motivation in sport and exercise: $\mathrm{A}$ review using the hierarchical model of intrinsic and extrinsic motivation. En R. N. Singer, H. A. Hausenblas, \& C. M. Janelle (Eds.), Handbook of sport psychology (2a ed., pp. 389-416). New York: John Wiley and Sons.

Vlachopoulos, S. P., Katartzi, E. S., Kontou, M. G., Moustaka, F. C., \& Goudas, M. (2011). The revised 
perceived locus of causality in physical education scale: Psychometric evaluation among youth. Psychology of Sport and Exercise, 12, 583-592.
Zahariadis, P., Tsorbatzoudis, H., \& Grouios, G. (2005). The Sport Motivation Scale for children: Preliminary analysis in physical education classes. Perceptual and Motor Skills, 101, 43-54.

Cuestionario de motivación en Educación Física en el tercer ciclo de educación primaria (CMEF:EP)

Indica hasta qué punto las frases se corresponden a lo que sientes, usando la escala desde totalmente desacuerdo (1) hasta totalmente de acuerdo (5).

Yo participo en las clases de Educación Física...

1. Porque la Educación Física es divertida

2. Porque puedo aprender habilidades que podría usar en otras áreas de mi vida

3. Porque me siento mal si no participo en las actividades

4. Porque está bien visto por el profesor y los compañeros

5. Pero no comprendo por qué debemos tener Educación Física

6. Porque esta asignatura me resulta agradable e interesante

7. Porque valoro los beneficios que puede tener esta asignatura para desarrollarme como persona.

8. Porque me siento mal conmigo mismo si falto a clase

9. Porque quiero que el profesor/a piense que soy un/a buen/a estudiante

10. Pero realmente siento que estoy perdiendo mi tiempo con esta asignatura

11. Porque me lo paso bien realizando las actividades

12. Porque, para mí, es una de las mejores formas de conseguir capacidades útiles para mi futuro

13. Porque quiero que mis compañeros/as valoren lo que hago

14. No lo sé; tengo la impresión de que es inútil seguir asistiendo a clase

15. Por la satisfacción que siento al practicar

16. Porque esta asignatura me aporta conocimientos y habilidades que considero importantes

17. Para demostrar al profesor/a y compañeros/as mi interés por la asignatura

18. No lo sé claramente; porque no me gusta nada

Factores e ítems asociados

Intrínseca:1, 6, 11, 15

Identificada: $2,7,12,16$

Introyectada: 3 y 8

Externa: 4, 9, 13, 17

Desmotivación: $5,10,14,18$ 